\title{
Cloud-Based Platform Solution for 5G Heterogeneous Networks
}

\author{
Kire Jakimoski, Oliver Iliev, Vladimir Bogoevski and Dejan Kochov \\ FON University, Faculty of Informatics, Skopje, Republic of Macedonia \\ kire.jakimoski@fon.edu.mk; oliver.iliev@fon.edu.mk
}

\begin{abstract}
The future of mobile communications is expected to be very diverse from the way it works today. Researches in $5 G$ wireless networks are actively developing and improving nowadays technologies. To assist the enormous mobile data, a huge amount of very small cells are expected to be deployed everywhere, including buildings and indoors, providing progress in the heterogeneous networks, which is considered as the main method towards $5 G$. Also, the $5 G$ air interface and spectrum should be linked together with LTE and Wi$\mathrm{Fi}$ to provide universal high-rate coverage. There are three technologies, mobile computing, wireless networks, and cloud computing that are converging into a field of Mobile Cloud Computing. With such massive heterogeneous networks, providers will face many difficult challenges in terms of procedure and administration, tiny cell deployment in economical manner, and intercell interference mitigation. Hence, cloud-based platform solution is great challenge for heterogeneous mobile and wireless networks in $5 G$.
\end{abstract}

Keywords: Cellular network, 5G, HetNets, Cloud computing, Changes, Improvements

\section{Introduction}

The present generation of mobile networks still transforms the way how people communicate and how information is accessed. Additionally, advancing and implementing technologies that enable true human-centered and connected devicedriven networks will come to redefine end user mobility along with the entire landscape of the global telecoms industry. 5G will imply great improvement in the importance of mobile access for realizing total ICT network advance and growth. Additionally, any mobile application and service will have the capability to connect to anything at any time - from people and communities to devices, processes, timely relevant information and commodities of all kinds in completely flexible, reliable and secure ways. Main expectations of $5 \mathrm{G}$ are: to increase the capabilities of what mobile networks can do, and to enhance upon what services they can distribute.

Enormous growth in mobile data traffic volume is verified, which is about two times larger every year. According to Cisco, Visual Networking Index (VNI) [3], the mobile data traffic size is predicted to grow at a Compound Annual Growth Rate (CAGR) of $61 \%$ from 2013 to 2018. The two major influences accounting for the growth in mobile data traffic are: the expansion of devices and development of applications that requires more data traffic. It is predicted by Qualcomm that 25 billion devices will be associated by year of 2020, due to the enormous growth of smartphones, Internet of things, Internet of Vehicles [4]. On the other hand, the applications that require more data, such as high resolution image transfer and video streaming, blow the data traffic. Additionally, varied types of applications have different quality of service demands. Therefore, wireless networks are upon great challenges to provide such a huge data flow and meet the customer's service demands [5]. 5G wireless network is expected to manage successfully the enormous data challenge and administer a throng of applications with diverse quality of service requirements. It is predicted to be a fully associated network, granting many 
quality of service to everyone and everything, at any time and everywhere. The capacity should be about a thousand times larger than the $4 \mathrm{G}$ capacity, latency low to 1 millisecond, and high energy and cost efficiency. Due to the necessity and usefulness of $5 \mathrm{G}$, industry and government agencies established the research around the world to assist the development of 5G. To meet the data challenge, the major and key role is network densification. Massive deployment of a small cells, such as microcell, picocell, femtocell, relay nodes, and Wi-Fi access points, which are lowpowered radio access nodes and have smaller coverage areas compared with microcells, is a crucial approach to enhance the network capacity, coverage performance, and energy efficiency. This densification approach has been used in nowadays wireless mobile networks particularly in 3G and 4G LTE systems, which basically results in a multi-tier mobile HetNet. Network operators are facing challenges as a result of the density distribution of small cells in a very large scale, and the improved level of complexity connected with decreased control over applications and data. It is 1) delicate to manage and control; 2) costly to densely setting up small cells; and 3) inter cell involvement. These factors are affecting and limiting the development of $5 \mathrm{G}$.

In this paper, to overwhelm the above problems, cloud based platform solution is presented, where small cells are associated to the cloud for administration and management. With the cloud based platform solution, the process and support can be easily accomplished. Integrated management expedites continuous development of the network, authorizing the network administrator to efficiently use network resources to support diverse applications while alter to the changing service requirements. Three main applications are considered: process and support, cloud aided business model for small cell distribution, and cloud aided interference management.

\section{Evolution of 5G}

In the development of $5 \mathrm{G}$ network there are three main approaches: spectrum enlargement, spectrum effectiveness improvement, and improving density of the network [2].

\subsection{Spectrum Enlargement}

To satisfy the enormously required data traffic and diversity of quality of service, it is fundamental to handle more spectrum by means of spectrum enlargement. Particularly, the low spectrum with ability easily to penetrate and signal diffusion properties, e.g., the TV whitespace starting from $570 \mathrm{MHz}$, to around $700 \mathrm{MHz}$, can be handled to boost the building penetration granting a greater coverage for connectivity. The IEEE 802.11 family of technologies experience large growth, with standards IEEE 802.22 for wireless regional networks and IEEE 802.11af for wireless local networks it's defined the route how to handle TV whitespace. Spectrum in high frequency bands have greater bandwidths and can support higher data rates, such as spectrum around $3.5 \mathrm{GHz}$ or millimeter wave frequency spectrum around $60 \mathrm{GHz}$. Additionally, because of the large path loss, high spectrum bands are well adapted to the small cells with brief communication scope. Millimeter wave can be of purpose for outdoor point-to-point backhaul links or for aiding indoor high-speed wireless apps. Actually, millimeter wave technologies have already been standardized for low-range services in IEEE 802.11ad. Still, these frequencies have not been completely analyzed for cellular applications. Possible reasons are the high propagation loss, penetration loss, rain declining and these frequencies are quickly absorbed or scattered by gases. Further growth are expected with use of the 
unlicensed spectrum or open spectrum integration which is more than necessary to mobile broadband networks. Basically it was used for simple offload onto Wi-Fi networks, but now, Wi-Fi networks are becoming more strongly integrated into mobile networks. There are also efforts underway to use LTE in unlicensed spectrum. The open spectrum boosts the capacity in two ways. Initially, a huge amount of spectrum is available across the $2.4 \mathrm{GHz}$ and $5 \mathrm{GHz}$ bands, with the 3.5 $\mathrm{GHz}$ band including larger spectrum in the future. And secondly, the open spectrum is mainly used in narrow coverage areas, with results in high-frequency reuse and much higher throughput rates per square meter of coverage versus typical cellular deployments.

The advantages of this success are offered hotspot services by cellular operators in public areas including airports, restaurants, shopping malls, hotels. The unification between mobile broadband and Wi-Fi networks can be either loose or tight. Loose unification means data traffic routes straightly to the Internet and reducing traversal of the operator network, named as "local breakout". Tight unification means data traffic, may cross the operator core network. Even though offloading onto Wi-Fi can scale down the traffic on the core network, the Wi-Fi network does not always have more unused capacity than the core network. The objective of future integrated mobile/Wi-Fi networks is to wisely merge the balance between the two. Unloading the data intelligently with success and granting users proper experience mandates measures such as automatically arranging user devices with the required Wi-Fi configuration options and automatically verify users on supported public Wi-Fi networks.

\subsection{Spectrum Effectiveness Improvement}

The capacity of the network can also be boost by improving spectrum effectiveness. To succeed in these improvements, diverse wireless technologies have been continuously developing to improve the spectrum efficiency. Basically many technical challenges of the future 5G HetNet will be surpassed with the use of massive MIMO and millimeter wave technologies and they can smoothly be unified with the current networks and access technologies. The deployment of massive MIMO, setting up many small antennas at the transmitter and receiver it can greatly boost the spectral and power effectiveness of the wireless network.

2.2.1. Massive MIMO - Massive MIMO: is basically composed of a few hundreds of low-cost antenna segments by employing large number of various antennas at the base station and the user unit. The technology either handle signals commuting between multiple paths in the environment or does beam-steering, in which multiple antennas concentrate their transmission energy in specific directions and therefore increase the throughput and enhance energy efficiency significantly. Additionally, it can also aid simultaneous transmissions to handle multiple users at the same time. Basically, with the deployment of large number of antennas on the transmitter and receiver there is enhancement of the overall performance of a wireless system. This performance enhancement is gained when the channel coefficients corresponding to different transmit-receive antennas experience independent fading. With massive MIMO, the capacity of the network can be improved by 10 times or more and the emitted energy-efficiency can be increased up to 100 times.

Some deployments of LTE are using 4x2 MIMO on the downlink (four base station emitting antennas and two mobile receiving antennas). LTE specifications incorporate greater order configurations, such as 4x4 MIMO, 8x2 MIMO. Engineers are now testing technology which is named massive MIMO systems, handling a far larger number of antenna components at the base station - 64, 128, and eventually even more. Usage in $5 \mathrm{G}$ of centimeter wave and millimeter wave bands, with their 
short wavelengths, will simplify massive MIMO, but even before then, 3GPP is considering massive MIMO for $4 \mathrm{G}$ systems a technique that improve the overall network efficiency as they allow mobile systems to support a huge number of UEs using multiuser MIMO techniques, this is called full-dimension MIMO (FDMIMO).

2.2.2. Cognitive Radio: Cognitive radio (CR) has been considered as an effective technique to improve spectrum efficiency by allowing unlicensed users to access the free spectrum opportunistically. Two main examples to effectively handle the spectrum are spectrum sensing and spectrum database. In the past, unlicensed users felt the spectrum to identify the availability of channels before transmission, and access the channels only when idle. In the future, unlicensed users can obtain the usability of channels through spectrum databases before entering the channels. Cognitive radios are constructed in order to grant highly reliable communication for all users of the network, always and everywhere needed and to simplify effective handling of the radio spectrum.

2.2.3. Device-to-Device Communication: With the development of Device-toDevice communication it is provided for proximity devices to interact with each other straightly without sending data to the base station or the core network. Compared with current cellular network communication, only half of the resources are required in direct communication. Therefore, it can considerably improve the spectral efficiency, because the communication is transported between two adjacent nodes, broadcast power can be saved. Additionally, this type of communication can lower delay, which is beneficial for latency-sensitive applications.

The initiation of direct device-to-device communication for LTE was introduced during 3GPP. However, the device-to-device communication functionality at the time was limited, basically concentrating on communication for emergency calls. D2D communication should be considered as a more general tool that is an integrated part of the overall wireless-access solution. Mainly, the D2D communication should be considered with the start of the development of 5G, instead of being lately presented as an add-on. Under network control, D2D communication can be carried out in licensed spectrum. However, the communication should also be possible in cases where there is no network coverage available, in which the D2D link has to be possible to establish without network control.

Models of applications involve social matching like detecting friends and family, push advertising for related notices, tourist notifications, venue services, crime alerts, communications between public safety staff in emergency events even when the rest of the network is unavailable, home automation, vehicle-to-vehicle communication, and detecting children leaving the proximity of their homes. As a new means of communicating, proximity services could result in innovative types of applications.

\subsection{Network Densification}

Few years ago, increasing the number of cell locations has been the main method for expanding capacity, granting gains far larger than what can be achieved by development in spectral effectiveness alone. The next wave of densification is by using what the industry calls "small cells". Central to small cell bolster is the heterogeneous network architecture, with various kinds of cells serving a coverage area, varying in frequencies used, radius, and even radio technology that is used.

Network densification via densely setting up cells of various types such as femtocells, picocells, microcells, and macrocells is a fundamental method to boost the capacity of the network, coverage performance and energy effectiveness. This 
way of densification is accepted in currently wireless cellular networks, in $3 \mathrm{G}$ and 4G networks, which basically results in a multi-tier cellular HetNet. In comparison with spectrum enlargement and spectrum effectiveness improvement, network densification is considered as the key technique to boost the capacity. This can be completed by massively deploying small cells, such as microcell, picocell, femtocell, which take the access points nearer to users so that network capacity can be greatly expanded and the latency can be decreased. The data traffic that comes from indoor users can also be transferred to $\mathrm{Wi}-\mathrm{Fi}$, such as the next generation $\mathrm{Wi}$ Fi 802.11ac, which is expected to support multi-gigabit data transmission rate. Such multi-tier networks with a diversity of radio access technologies are specified as heterogeneous networks.

\section{Challenges of Heavy Distribution of Small Cells}

Despite the important role HetNets have for $5 \mathrm{G}$, the enormous distribution of small cells can cause serious challenges for network operation and administration, deployment and so on.

\section{A. Network Distribution}

Non-tech savvy users can have many problems with distributing their small cells. In addition improper configuration or installation done by consumers can result in bad impact on the existing systems. Furthermore, even though the users can distribute few small cells for their own needs, the network administrator however needs to deploy a huge number of small cells, which can be greatly challenging due to some of these reasons: i) network administrators are on a narrow budget because they are always buckling under the strain of gradually adding network infrastructures; ii) the enormous distribution of small cells is expensive for network operators, when it comes to operating and capital expense, like site lease, installation prices, additional costs for electricity and backhaul. For that reason, the problem of how to distribute small cells heavily in an easy and expense-efficient way needs to be cautiously addressed.

\section{B. Administration and Application}

With a dense number of small cells, network administration and management can become more complex, as it will require human effort to set up, configure, auditor, and cultivate the small cells. Furthermore, the traffic may vary often, depending on location and time; it is hard for the network administrator to smoothly consume the network resources by managing the small cells at various sites for adapting to the dynamic demands. Additionally, small cells may be switched ON/OFF or replaced at any time, which can make optimization and administration of the network really challenging.

\section{Intercell Interference Mitigation}

As the network density grows, inter-cell interference is expected to arise, which undoubtedly limits the boost obtained from massively distributed small cells. In HetNets, intercell tampering is likely to happen in various scenarios. For instance, the macrocell can cause serious conflict to the users in the small cell and the consumers of macrocell may gain interfering signal from the adjacent small cells. The interference is mirrored in inferior signal to interference plus noise ratio (SINR) that can weaken the efficiency and the consumer experience. Without mitigating conflict among distant cells, HetNets can't be successfully distributed. One way to manage the intercell interference is spectrum dividing, where the entire spectrum is 
split into distinct parts for various cells. Nevertheless, it can point to inefficient spectrum consuming. Intercell Interference Coordination (ICIC) is imported in $3^{\text {rd }}$ Generation Partnership Project (3GPP) release 8 to mitigate interference.

Almost blank subframes (ABS) are part of Enhanced Inter-Cell Interference Coordination (eICIC) framework that the $3 \mathrm{GPP}$ has proposed as means to contest excessive co-channel cross-tier interference in heterogeneous network (HetNet) scenarios. The ABS concept is established on blanking some subframes of the interferer tier and scheduling the notably exposed UEs in these subframes. The exposed users thus get part of radio resources where cross-tier interference is lower. The ABS are called almost blank because not all resource components are allowed to be blanked. The cell-specific reference symbols that are used for radio resource management (RRM) measurements and channel estimation have to stay present. The ABS procedure uses time domain orthogonalization in which explicit sub-frames are left blank by the unbiased BS and off-loaded users are scheduled within these subframes to avoid inter-tier interference. This improves the overall throughput of the off-loaded users by sacrificing the time sub-frames and throughput of the unbiased BS. The larger bias values result in higher degree of offloading and thus require more blank subframes to protect the offloaded users. With a particular number of ABSs or the ratio of blank over all of the sub-frames (i.e., ABS ratio) that ensures the minimum throughput of the unbiased BSs, this criterion allows a user to select a cell with maximum ABS ratio and may even correlate with the unbiased BS if ABS ratio diminish significantly. In order to assist pico downlink transmissions, the macro eNodeBs can mute all downlink transmissions to its UEs in ABS. These subframes are called "almost blank" because a macro can still transmit some broadcast signals over these subframes. Since these broadcast signals only occupy a small fraction of the OFDMA subcarriers, the overall interference a macro causes to a pico is much less during these ABS periods. Thus, the pico can transmit to its UEs at a much higher data rate during ABS periods. Note that, a pico is also allowed to transmit to its UEs during non-ABS periods. This could provide good enough performance to UEs very close to the pico. An example of ABS schedule is shown in Figure 1 [7].

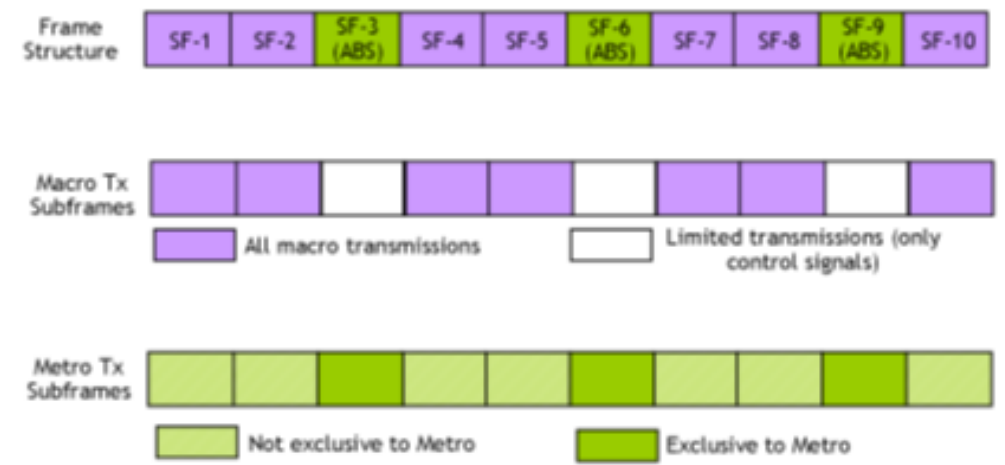




\section{Requirements of 5G Wireless Networks}

The standard for the $5 \mathrm{G}$ wireless network still isn't set. We can expect to know the precise technical specifications of this network in the near future. Nevertheless, the accepted technical requirements by the industries and academia are the following [1-5].

Coverage and data rate - It is expected that users will experience a minimum data rate of $1 \mathrm{~Gb} / \mathrm{sec}$, and have connectivity anytime and anyplace. Naturally, as the low mobility UEs channel shifts very slower than the high mobility ones, those UEs need extra resources for CSI acquisition. For that reason, the peak data amount needed by high and low mobility consumers in the 5G network may vary. The network should further insure a certain QoS for users travelling with great speeds (e.g. high speed trains that reach a speed of $500 \mathrm{~km} / \mathrm{hr}$ or planes) where the current network can't competently support.

Scalability - The future $5 \mathrm{G}$ network is predicted to incorporate enormous load of connected devices that can reach up to 100 times that of the present wireless network. The most promising use cases in this matter are wearable computing, machine type communications, wireless sensors, and internet of things. Generally, these connected machines can have distinct requirements in terms of communications rate, delay and dependability. The 5G network needs to be capable to scale adequately when it comes to signaling traffic, like authentication/authorization for huge numbers of IoT devices. When it comes to supporting combination of IoT apps and more classic services like video and voice, the term "scalability" has another dimension. 5G networks are supposed to be capable to support both huge-data-rate/low-latency common services alongside device to device applications that need much less bandwidth.

Network Flexibility - The 5G network structure need to allow the RAN and the main network to develop and adjust separately of one another. Making an adjustment/modification of one should not mandate adjustments/modification of the other. For this to work, the RAN and the packet core need to dodge shared dependencies. Furthermore, 5G networks need to support multi-RAT connection efficiently and effectively. This provides the capability to:

- Implement an access-agnostic packet core across different radio technologies (e.g., cellular, Wi-Fi) to support reliable authentication, session stability and security.

- Implement plug-and-play capability where a new access technology can be attached to the packet core without any adjustments.

Decoupling the packet core from the RAN can also help to further clarify the network. Flattening upgrades, the network scalability by allowing some operations to be pushed towards the user/edge.

Security - Public-security networks can have bigger insurance requisites then commercial networks, including physical safety of data centers, core sites, and cell sites. When in fact LTE networks doesn't have to encode traffic in backhaul and core networks, public-safety applications can select to encrypt all IP traffic using virtual private networking approaches. Concerning the role of $5 \mathrm{G}$ networks as critical infrastructure, an agreement must be made on just how critical these should be, because expanding criticality arrives with a price tag in terms of security. The accepted assurance for IT products is Common Criteria (ISO 15408). If $5 \mathrm{G}$ is going to be a common accepted platform for the Networked Society vision, it appears that Common Criteria agreement can enter as an extra security requirement besides SECAM. Nevertheless, the impact will not stop there. Let's consider the scenario where vehicle/road safety is reliant on $5 \mathrm{G}$ network security. What does this actually 
mean? These days, safety-related vehicles systems need to follow certain comprehensive standards, like ISO 26262 . If $5 \mathrm{G}$ security grows into being a crucial bond in the control circle of all these applications, does it mean that $5 \mathrm{G}$ networks should be approved by all these standards?

In essence, the 5G network is planned to be a platform for a wide range of consumer groups and applications, that doesn't unquestionably mean that it is essential for the $5 \mathrm{G}$ network to bear all security responsibility and relevant costs. However, there is a big chance that there will be a lot overlapping agreement requirements; it is understandable that abundant expenses will be induced on $5 \mathrm{G}$ network products. Whether or not these expenses will prevent $5 \mathrm{G}$ from providing beneficial services to these applications, and there are basically two different methods to manage this security overload on the $5 \mathrm{G}$ network.

First, the approach of network sharing may be an essential tool to manage the various requirements of different applications and user groups. Slicing is rarely seen as a way to afford private sub-networks, each developed for specific types of traffic specifications. One such characteristic may be associated to security and safety demands. By having a correctly imported, high-assurance isolation mechanism to support sharing, it will be likely to constrain the effect of security requirements to single slices, rather than the whole network. The expenses of high safety and certification can accordingly be linked into a framework virtualization/isolation layer.

Secondly, we have the choice to "remove" security demands from the $5 \mathrm{G}$ network shares by simply putting the accountability in the endpoints; in different words, in connected devices or organization data centers. Data security is a model of a service that could be managed this way.

To summarize, the fact that $5 \mathrm{G}$ is made to be a platform for a wide range of new consumer groups and applications does not automatically mean that it is necessary (or even desirable) for the 5G network to carry all safety necessarily and related expenses. However, 5G networks evidently can supply some highly valuable safety services. In addition the isolation/slicing itself, many other examples of networkenabled security as a service will be attractive to multiple user groups, including network enforced security policies, authorization, key management and data security services.

Associated standardization activities - The Next Generation Mobile Networks (NGMN) Alliance is a cellular telecommunications association of cellular operators, vendors, producers and research centers. The project results from this alliance have been accepted by 3 GPP and IEEE. Few years ago, the NGMN Alliance released the launch of a global initiative for 5G, with the goal of managing the evolution of technologies and standards to meet the requirements of the future. In this inception stage, the NGMN Alliance has delineated the concerns for $5 \mathrm{G}$ in conditions of user experienced data rate, latency, mobility and so on. Because HetNets are fundamental part in 5G evolution, 3GPP standards intent to guide the operation and management of small cells. Particularly, inter-cell interference coordination (ICIC) was brought in 3GPP Release 8 in which base stations can communicate with each other through the X2 interface. Enhanced ICIC (eICIC) was brought in 3GPP Release 10, which unified Almost Blank Subframes (ABS) to reduce the interference in time domain. In 3 GPP Release 11, ICIC is developed to additional enhanced ICIC (feICIC). In 3GPP Release 12, systems for effective activity of the small cell layer that were acquainted, such as interference reduction through optimally powering small cells. Additionally, HetNet flexibility, Wi-Fi mobile cooperation, D2D application, selfoptimizing network are included in 3GPP Release 12. 


\section{Heterogeneous Network and Cloud Association}

Cloud computing is becoming progressively imperative in today's corporations because of the benefits provided, such as better adaptability, improved security, scalability and lower costs. To focus on the challenges stated in part 3, a cloud based architecture and association with the heterogeneous networks solution is presented, that handle the cloud as regulation and management of the network. Also with the mobile cloud computing technology, 5G networks supported by the cloud are making availability for the computer applications including data processing. Despite the access potentiality with $5 \mathrm{G}$ mobile networks as well as high data rate, low latency and high volume for various applications, there is a demand to satisfy mobile users' requirements from the quality of experience (QoE) point of view, such as motion-aware applications [6]. The intention is to invoke a unique style of applications enabled by emotion-aware mobile cloud computing identifying user's emotional changes.

With cloud association, the installation, control, management, and upgrade can be quickly performed. Additionally, the unified management simplifies the optimization of the network that is helpful for the network provider to make effective use of the network resource to satisfy various QoS demands and adapt to the changing requirements. A comparable approach is cloud radio access network (C-RAN), which allocates radio head ends at various sections and merges the baseband processing components in the cloud. Diversely from C-RAN, in this cloud association the user's data is not handled by the cloud, alternatively the cloud is concentrate on operation, maintenance, and administration of small cells to grant functions by effectively using network resources. Even though HetNets are great substitute to supply seamless scope and immense volume in $4 \mathrm{G}$ systems, there are two significant challenges to block their profitable developments:

1) The SE efficiency should be improved as a result of the intra and inter-cell, Coordinate Multi-point (CoMP) need a great number of signaling in backhaul links to reduce interferences between HPNs and LPNs, which frequently results in the forced backhaul links.

2) The extremely heavy LPNs can make better capacity with the expense of consuming too much energy, which results in low EE efficiency.

Cloud radio access networks (C-RANs) are by now acknowledged to curtail the capital and running expenses, as well as to supply a high transmission bit rate with excellent EE efficiency. Cloud radio access networks (C-RANs) are by now known to decrease the capital and operating costs, as well as bring a high transmission bit rate with excellent EE performances. The remote radio heads (RRHs) act as soft replay by compressing and forwarding the received signals from UEs to the centralized baseband unit (BBU) pool through the wire/wireless fronthaul connection. To determine the benefits of C-RANs, the joint decompression and decoding schemes are executed in the BBU pool. Precisely, HPNs should be still critical in C-RANs to assure the backward compatibility with the existing cellular system and support smooth coverage since RRHs are mainly deployed to provide high capacity in special zones. With the help of HPNs, the multiple heterogeneous radio networks can be gathered, and all system control signaling are brought wherein. Therefore, we incorporate HPNs into C-RANs and thus H-CRANs are proposed to take full advantages of both HetNets and C-RAN, in which cloud computing capabilities are exploited to solve the aforementioned challenges in HetNets. 


\section{System Architecture of H-CRANs}

Likewise, with the traditional C-RAN, an extremely large number of RRHs with low energy consumptions in the proposed H-CRANs are agreed with each other in the streamlined BBU pool to accomplish high cooperative gains. Only the front radio frequency (RF) and clear symbol processing functionalities are implemented in RRHs, while the other important baseband physical processing and procedures of the upper tiers are executed jointly in the BBU pool. In a sequence, only incomplete functionalities in the PHY layer are incorporated in RRHs. Nevertheless, distinct from C-RAN, the BBU pool in H-CRANs is interfaced to HPNs for mitigating the cross-tier interferences between RRHs and HPNs through the centralized cloud computing based cooperative processing techniques. Additionally, the data and control interfaces between the BBU pool and HPNs are combined and denoted by S1 and X2, accordingly, whose definitions are inherited from the standardization definitions of 3rd generation partnership project (3GPP). As the voice service can be supplied neatly through the packet switch mode in 4G systems, the expected $\mathrm{H}$ CRAN can support both voice and data services both at the same time, and the voice service is preferred to be administered by HPNs, as long as the high-data packet traffic is for the most part served by RRHs. In comparison with the standard C-RAN architecture, the proposed H-CRAN alleviates the fronthaul needs with the participation of HPNs. Because all signals are centralized handled in the BBU pool for UEs combined with RRHs, the cloud computing based cooperative processing techniques inherited from the virtual MIMO can produce high variety and multiplexing gains. Likewise, with C-RANs, the inter-RRHs interfaces can be suppressed by the advanced cloud computing based large-scale cooperative processing techniques in the BBU pool. The cross-tier intrusion among HPNs and RRHs can be diminished through the cloud computing based cooperative RRM (CCCRRM) via the interface X2 between the BBU pool and HPNs. To achieve better EE performances of H-CRANs, the activated RRHs are adaptive to the traffic volume. When the traffic capacity is low, some probable RRHs fall into the sleep mode under administration of the BBU pool. Nevertheless, when the traffic load becomes huge in a small special zone, both the HPN with massive MIMO and dense RRHs work together to meet the tremendous capacity needs, and even the corresponding desired RRHs can borrow radio resources from neighboring RRHs.

The small cells are distributed thickly in the network, in indoor scenarios such as homes and offices, or in outdoor scenarios such as road crossroad, squares, and malls. The network provider can set up small cells with open and closed connection models. Controlling, configuration, optimization, and mobility control is integrated in the cloud. The integrated characteristic allows the network providers to control the network in a more effective way. Because the traffic requirements may greatly differ, the cloud aids the network providers to assign the resources on need and relate small cells effectively to grant services with consistent coverage, high data rates and low latency. The cloud can import the coming advantages to the wireless networks.

Easier maintenance and administration - It consolidate maintaining, administration, and troubleshooting of various sites from a single platform. It can add services such as remote audit, real-time reviewing, easing the configuration and device administration. With the cloud, the administrators and users can connect to the network data whenever they need and from wherever they are through the Internet.

Extensible - The cloud supported design can reduce the distribution by setting up small cells through the cloud to simplify the installation of small cells when network extensions are needed. 
Effectiveness - It allow the network administrator to swiftly re-allocate network resources to meet the variable and improvident user requirements. For example, in low demand hours, some small cells can be powered off, but they are powered on while high demand hours easily and quickly through the cloud. Additionally, is aiding optimization of the efficiency of the network and rationally adjust the network resources. This way the network administration is cost effective.

\title{
6. Conclusion
}

If the expectations are fulfilled for the future technologies $5 \mathrm{G}$ will change the way networking works. The capacity of $5 \mathrm{G}$ wireless connection should be expanded massively far above the earlier generations in order to support large number of applications. As stated by the ITU, there should be more than 9.2 billion mobile users worldwide by the year of 2020, with large increase in subscriptions to device-to-device and Internet of Things. The main 5G potentials are much bigger data rates, reduced latency and extreme reliability. To make these potentials real the traffic of 5G should have enormous growth in an inexpensive way, indicating a need for a tremendous reduction in the cost and energy consumption. A flexible network solution framework will become an essentiality for consideration of both LTE and air interface evolution, cloud will alter the whole mobile ecosystem, and 5G will speed up the development of large-scale services and applications.

\section{References}

[1] Rysavy Research/4G Americas, "Beyond LTE: Enabling the Mobile Broadband Explosion", August 2015.

[2] 4G Americas, "5G Technology Evolution Recommendations", October 2015.

[3] Cisco, "Cisco visual networking index: Global mobile data traffic forecast," White Paper, 2014.

[4] N. Lu, N. Zhang, N. Cheng, X. Shen, J. W. Mark, and F. Bai, "Vehicles meet infrastructure: Toward capacity-cost tradeoffs for vehicular access networks," IEEE Transactions on Intelligent Transportation Systems, vol. 14, pp. 1266 - 1277, 2013.

[5] X. Zhang, W. Cheng, and H. Zhang, "Heterogeneous statistical quality of service provisioning over 5g mobile wireless networks," IEEE Network, vol. 28, pp. 46-53, 2014.

[6] Mugen Peng, Senior Member, IEEE, Yuan Li, Jiamo Jiang, Jian Li, and Chonggang Wang, Senior Member, IEEE, "Heterogeneous Cloud Radio Access Networks: A New Perspective for Enhancing Spectral and Energy Efficiencies", 11 Oct 2014.

[7] Algorithms for Enhanced Inter Cell Interference Coordination (eICIC) in LTE HetNets Supratim Deb Pantelis Monogioudis, Jerzy Miernik, James P. Seymour Feb 2013.

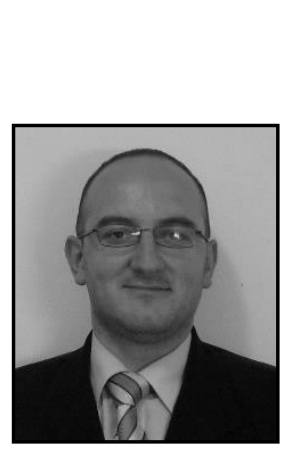

\begin{abstract}
Authors
Kire Jakimoski, he received his B.Sc. degree in the field of Telecommunications from the Military Academy "Mihailo Apostolski" in Skopje, R. Macedonia in 2002, M.Sc. degree in Electrical Engineering in the field of Telecommunications from the Ss. Cyril and Methodius University in Skopje, R. Macedonia in 2007, and Ph.D. in technical sciences from the Ss. Cyril and Methodius University in Skopje, R. Macedonia in 2013. From 2002 to 2006 he works as an Officer for Telecommunications in the Ministry of Defense in the Republic of Macedonia. From January, 2006 to March, 2012 he works as an adviser for information security in the Directorate for Security of Classified Information in the Republic of Macedonia. From March, 2012 he is with the Faculty of Informatics, FON University in Skopje. Also, he is an author/co-author of around 40 published research papers and one book. He is an Assistant Professor and Vice Dean at the Faculty of Informatics, FON
\end{abstract}


University in Skopje, Macedonia. His research interests include Wireless and Mobile Networks, Heterogeneous Wireless Networks, Computer Networks, Digital Telecommunications, Information Security.

Oliver Iliev is a Full Professor at the Faculty of Informatics, FON University, Skopje, Republic of Macedonia.

Vladimir Bogoevski and Dejan Kochov are students at the Faculty of Informatics, FON University, Skopje, Republic of Macedonia. 